\title{
Initial Trustworthiness Perceptions of a Drone System based on Performance and Process Information
}

\author{
Theodore Jensen \\ Department of Computer Science \& \\ Engineering \\ University of Connecticut \\ Storrs, Connecticut \\ theodore.jensen@uconn.edu \\ Ross Buck \\ Department of Communication, \\ University of Connecticut \\ Storrs, Connecticut \\ ross.buck@uconn.edu
}

\author{
Yusuf Albayram \\ Department of Computer Science \& \\ Engineering \\ University of Connecticut \\ Storrs, Connecticut \\ yusuf.albayram@uconn.edu \\ Emil Coman \\ Health Disparities Institute, \\ University of Connecticut Health \\ Center \\ Hartford, Connecticut \\ coman@uchc.edu
}

\author{
Mohammad Maifi Hasan Khan \\ Department of Computer Science \& \\ Engineering \\ University of Connecticut \\ Storrs, Connecticut \\ maifi.khan@uconn.edu
}

\author{
Md Abdullah Al Fahim \\ Department of Computer Science \& \\ Engineering \\ University of Connecticut \\ Storrs, Connecticut \\ md.fahim@uconn.edu
}

\begin{abstract}
Prior work notes dispositional, learned, and situational aspects of trust in automation. However, no work has investigated the relative role of these factors in initial trust of an automated system. Moreover, trust in automation researchers often consider trust unidimensionally, whereas ability, integrity, and benevolence perceptions (i.e., trusting beliefs) may provide a more thorough understanding of trust dynamics. To investigate this, we recruited 163 participants on Amazon's Mechanical Turk (MTurk) and randomly assigned each to one of 4 videos describing a hypothetical drone system: one control, the others with additional system performance or process, or both types of information. Participants reported on trusting beliefs in the system, propensity to trust other people, risk-taking tendencies, and trust in the government law enforcement agency behind the system. We found that financial risk-taking tendencies influenced trusting beliefs. Also, those who received process information were likely to have higher integrity and ability beliefs than those not receiving process information, while those who received performance information were likely to have higher ability beliefs. Lastly, perceptions of structural assurance positively influenced all three trusting beliefs. Our findings suggest that a) users' risktaking tendencies influence trustworthiness perceptions of systems, b) different types of information about a system have varied effects on the trustworthiness dimensions, and c) institutions play an important role in users' calibration of trust. Insights gained from this study can help design training materials and interfaces that improve user trust calibration in automated systems.
\end{abstract}

Permission to make digital or hard copies of all or part of this work for personal or classroom use is granted without fee provided that copies are not made or distributed for profit or commercial advantage and that copies bear this notice and the full citation on the first page. Copyrights for components of this work owned by others than ACM must be honored. Abstracting with credit is permitted. To copy otherwise, or republish, to post on servers or to redistribute to lists, requires prior specific permission and/or a fee. Request permissions from permissions@acm.org.

HAI '18, December 15-18, 2018, Southampton, United Kingdom

(C) 2018 Association for Computing Machinery.

ACM ISBN 978-1-4503-5953-5/18/12 ..\$15.00

https://doi.org/10.1145/3284432.3284435

\section{CCS CONCEPTS}

- Human-centered computing $\rightarrow$ Collaborative interaction; HCI theory, concepts and models; Empirical studies in HCI;

\section{KEYWORDS}

human-automation trust; trusting beliefs; perceived trustworthiness; initial trust

\section{ACM Reference Format:}

Theodore Jensen, Yusuf Albayram, Mohammad Maifi Hasan Khan, Ross Buck, Emil Coman, and Md Abdullah Al Fahim. 2018. Initial Trustworthiness Perceptions of a Drone System based on Performance and Process Information. In 6th International Conference on Human-Agent Interaction (HAI '18), December 15-18, 2018, Southampton, United Kingdom. ACM, New York, NY, USA, 9 pages. https://doi.org/10.1145/3284432.3284435

\section{INTRODUCTION}

Recent advancement in computing technologies has opened up the possibility of next-generation cyber-human systems (e.g., multiUAV supervisory control, remotely operated air and ground vehicles, robot-assisted emergency response systems) where users will be required to interact and cooperate with autonomous or semiautonomous systems to accomplish challenging and risky tasks (e.g., surveillance, battlefield operation, navigation). Prior work has noted that inappropriate levels of user "trust" in such safety-critical systems, including both overtrust and undertrust, can have undesirable and even fatal consequences [35], calling for further research on how to achieve appropriate "trust calibration." Characteristics of the human operator, the environment, and the automated system have been identified as factors that can influence trust in an automated or autonomous trustee. Hoff and Bashir suggested a three-layered model in which these factors contribute to dispositional, situational, and learned trust, respectively [19].

Interestingly, while a significant amount of work has investigated these aspects of trust in the context of human-machine interactions, very little is known regarding "initial trust" in this context. Initial trust, or trust in an unfamiliar party [30], contributes to the "risktaking in relationship" that defines later trust $[2,27]$. Moreover, first 
impressions and system training have been found to affect later trust and reliance in safety-critical systems $[6,13]$.

To complement prior efforts focusing on trust during humanmachine interactions, the present study focuses on factors that contribute to the development of initial trust. Specifically, we aim to observe how characteristics of the operator (i.e., dispositional), perceptions of the institution behind a system (i.e., situational), and information about a system (i.e., learned) influence initial trustworthiness perceptions of that system. Toward this, we designed 4 narrated videos with different information about a hypothetical drone system. We recruited 163 naive participants (i.e., without prior experience with drone systems) on Amazon MTurk, randomly assigned each to one of the 4 video groups, and asked them to imagine that they were going to operate the drone in a safety-critical task. Given the sample's lack of exposure to similar technologies, we can observe how system information contributes to learned trust, and how dispositional and situational factors simultaneously influence trust evaluations.

After watching their video, participants rated statements on trusting beliefs in the system's ability, integrity, and benevolence, and institutional trusting beliefs in situational normality and structural assurance. They also reported on demographics, propensity to trust other people, and risk-taking tendencies. We discuss the implications of our findings for human-computer trust research and system design.

\section{RELATED WORK}

\subsection{Trust}

The concepts of "trust" and "trustworthiness" have been extensively studied by various research communities such as psychologists and communication theorists in the context of human-human interactions. While some refer to it as a behavioral state, an individual disposition, a set of social expectations, or an emotion, there is consensus that risk lies at the heart of trust $[1,5,9,38]$. Mayer et al.'s model gives one of the most widely accepted definitions of human-human trust: "The willingness of a party to be vulnerable to the actions of another party based on the expectation that the other will perform a particular action important to the trustor, irrespective of the ability to monitor or control that party" [27].

In this relationship between trustor and trustee, trust is affected by characteristics of the trustor, the trustee, and the situational context [27]. A trustor's trusting beliefs contribute to their trust, realized as risk-taking in the relationship with the trustee. These beliefs are perceptions of a trustee's trustworthiness, which Mayer et al. operationalize into three characteristics: ability, integrity, and benevolence. Ability relates to the trustee's skills or competencies within some domain. Integrity reflects that the trustee "adheres to a set of principles" that is acceptable to the trustor. Benevolence relates the extent to which a trustee will do good for the trustor [27].

\section{2 “Trust” in Human-Machine Interactions}

Many researchers have applied insights from the human-human trust literature to study trust between a human and a machine, computer, or automated system. This notion is motivated by work in the "Computers are Social Actors" paradigm, which has shown that users apply various social norms to computer interaction partners.
These include perceptions of praise and derogation, treating different voices as distinct social actors, applying gender stereotypes, and using politeness [34]. In the latter study, participants worked with a computer and subsequently assessed its performance in one of three ways: on the same computer, with pencil and paper, or on a different computer [37]. Those in the same computer condition rated the computer as more friendly and competent than those in the other conditions, suggesting that politeness was employed when giving a direct assessment. Despite these responses, at the end of the experiment, participants denied acting polite toward the computer. Reeves and Nass suggest that these social responses occur "mindlessly" in that, even though they may think it's nonsensical, people apply the notion, "When in doubt, treat it as human," when interacting with computers [33, 37].

Among prior efforts that attempt to understand trust in the human-machine context, Bonnie Muir defined trust in humanmachine interaction as a function of expectations held by a member of a system regarding the persistence, technical competence, and fiduciary responsibilities from another member of the system [32]. More recently, in line with Mayer et al.'s human-human model, Hoff and Bashir noted characteristics of the human operator, the environment, and the automated system as factors influencing trust in an automated trustee. They suggest a three-layered model in which these factors contribute to dispositional, situational, and learned trust, respectively [19].

Hoff and Bashir suggest that age, gender, and personality differences are components of dispositional trust [19], while prior work has begun to investigate the factors influencing dispositional trust in automation [20].

Prior work has further identified situational normality and structural assurance as two aspects of institutional trust, where situational normality consists of beliefs about an institution's trustworthiness in a given context [25, 29], and structural assurance relates to a belief that guarantees or "safety nets" are in place to protect the user if something goes wrong $[25,29]$. These institutional perceptions constitute one aspect of Hoff and Bashir's situational trust.

Regarding learned trust (i.e., in a specific trustee), Lee and Moray refer to performance, process, and purpose as the bases for trust in automation [23]. Performance refers to the consistency and reliability of system behavior and its history of operation. Process relates to how the system operates and the degree to which its algorithms are appropriate in a given context. Purpose is the extent to which the system is being used within the "realm of the designer's intent” [23]. The lexical dissonance between Mayer et al.'s perceived trustworthiness characteristics and Lee and Moray's terms distinguishes human-human from human-computer trust. In general, a trustor is concerned with the what (ability or performance), the how (integrity or process), and the why (benevolence or purpose) of their interaction partner's behavior. Trust "calibration" occurs when a system user adjusts their perceptions of these characteristics to better fit a system's actual reliability [24]. While prior work on trust in e-Commerce and online recommendation agents [2, 22, 29-31] have operationalized perceived trustworthiness using ability, integrity, and benevolence items adapted from Mayer's research [26], to the best of our knowledge this approach has not been taken in the trust in automation literature. 


\subsection{Initial Trust and the Current Study}

"Initial trust," or trust in an unfamiliar party [30] has been shown to precede and contribute to the risk-taking in relationship that defines later trust [2,27]. First impressions and system training have been found to affect later trust and reliance in safety-critical systems as well $[6,13]$. Furthermore, research on organizational information systems and e-Commerce has found factors such as reputation and perceived usefulness to influence initial trust $[2,22,25,30]$. Despite the importance of initial trust, prior work on safety-critical systems has not explored this and generally observes trust during interaction $[3,10]$.

As such, to complement prior efforts, we aim to observe how characteristics of the operator, perceptions of the law enforcement agency behind the system, and information about a drone system influence initial perceived trustworthiness. We expect that, in addition to general propensity to trust others [39], individual differences in risk-taking are influential in trust evaluations of safety-critical systems. Furthermore, by measuring "institutional trust," we treat the system itself as a distinct trustee, and avoid the assumption that trust in the system is the same construct as trust in system designers or other members of the organization.

A main goal of this study is to observe whether users perceive an automated system to have ability, integrity, and benevolence, and the extent to which the aforementioned dispositional, situational, and learned factors contribute to these perceptions. The details of our study are presented in the following sections.

\section{METHODOLOGY}

The purpose of this study is to observe how information about a system's performance and process can be communicated to influence initial beliefs in the ability, integrity, and benevolence of a drone system, and how personal and institutional factors affect these perceptions. More specifically, this study seeks to answer the following research questions:

RQ1: Does a person's propensity to trust other humans translate to a computer trustee?

RQ2: Do a person's risk-taking tendencies influence their initial trust?

RQ3: How do performance and process information influence beliefs in a system's ability, integrity, and benevolence?

RQ4: What role does institutional trust play in trust of a computer system itself?

\subsection{Design of Videos}

To investigate the aforementioned research questions, we created 4 videos describing a hypothetical drone system.

We chose video as the mode of communication because there is evidence that videos can be effective in introducing a system to users (e.g., system training) by utilizing both visual and auditory information processing channels, which leads to higher engagement $[7,17,28,36,41]$. This method also allows us to isolate the effects of information participants receive from factors such as experience and interface features that can influence trustworthiness perceptions during use. We chose to investigate a drone system because most individuals are familiar with their safety-critical applications.

\begin{tabular}{cccc}
\hline Label & n & Link to Video & Length \\
\hline Control & 41 & https://youtu.be/DuMwSsrEG5s & $50 \mathrm{~s}$ \\
Performance & 39 & https://youtu.be/RJdwtSuGmAc & $71 \mathrm{~s}$ \\
Process & 39 & https://youtu.be/2BTbNTAG19A & $70 \mathrm{~s}$ \\
Perf-Proc & 44 & https://youtu.be/c5JrIdQNkY4 & $92 \mathrm{~s}$ \\
\hline
\end{tabular}

Table 1: List of the 4 videos used in the study, which can be viewed on YouTube. The original video can be found at: https://www.dvidshub.net/video/411919/ mq1b-predator-gcs-broll.

Information was given in the form of narration. The content was reviewed and revised by authors over several iterations to ensure clarity for our naive participants and relevance to Lee and Moray's definitions [23] of performance and process applied to our drone system. These two information types were defined as follows:

- Performance refers to the consistency and reliability of system behavior. The narrated performance information included how external/internal factors (e.g., poor network connections, software glitches) can influence the reliability of the system and what the consequences might be.

- Process details the qualities that govern system behavior, such as its algorithms. The narrated process information included how the system behaves to make it robust against possible failures (e.g., sensors are used to monitor flight stability).

Lee and Moray's third characteristic, the purpose of the system, was given in all videos. We determined this was necessary to give participants sufficient information to understand the system and their role as operator. Thus, the control group watched a video containing only "baseline" information (i.e., describing what the system was used for), and the three experimental groups watched a video containing the same baseline content followed by either performance or process information, or both. Table 2 shows the full narration transcript.

The video's visual content was taken from a publicly available video of drone operation. The original audio was replaced by narration recorded by one of the researchers. To investigate the effect of performance and process information alone, as well as their interaction, videos were trimmed to the length of their narration. This avoided both video playing without narration and repeated visual content. Longer videos therefore contain visual content that shorter videos do not. Because the video displays neutral images of operators at a control panel, we expect that the narration describing a safety-critical drone task was more salient. However, we acknowledge the potential effect of the visual content and refer the reader to Table 1 to view the videos on YouTube. While participants watched clear versions of the videos, we blurred out certain parts in the shared links for privacy reasons.

\subsection{Recruitment}

We posted the study as a Human Intelligence Task (HIT) on Amazon's Mechanical Turk (MTurk) service. Using MTurk's eligibility criteria, the HIT was made available to users 18 years or older, living in the United States, with at least 1000 completed HITs and a 95\% HIT approval rating. When participants accepted the HIT, 
Hello! The video you are watching presents a hypothetical scenario where operators are using a drone system to assist government law enforcement in stopping human traffickers. The system consists of an Unmanned Aerial Vehicle, or UAV, and a display that the operator observes while controlling the system. The operator is responsible for navigation of the drone and reporting locations of suspected human traffickers. Timely and accurate identification of violent criminals is extremely important as failures can put innocent civilians' and law enforcement officers' lives in danger. While the operators may shoot at targets if necessary, this is used only as the last option, as it could lead to hitting innocent civilians near the target or causing property damage.

While the system operates effectively most of the time, there can be occasional errors that impact video quality and drone maneuverability, caused by factors such as poor network connections and software glitches. As a result, operators may experience rare events such as screen blackouts or loss of connectivity lasting at most a few seconds.

To make the system robust against such failures, the UAV has on-board algorithms that use sensors to improve flight stability and maneuver-

Process ability. Information about system health is also automatically monitored and sent back to the operator over a network connection. This allows the operator to monitor and override system control if needed.

Table 2: Narration script for the videos. All videos contained
"baseline" information describing the purpose of the system,
while the Performance video additionally contained the per-
formance information, Process the process information, and
Perf-Proc both types of additional system information.

they were shown an information sheet and link connecting them to the study hosted on our university's Qualtrics server.

There were 3 pre-screening questions to prevent participants from guessing the eligibility criterion. Participants had to answer "No" to the question "Have you ever operated drones in the past?". This screened out individuals having operated recreational drones in addition to systems like that described in our study, ensuring that we observed initial trust. We did not disclose this eligibility criterion to any participant.

Ineligible participants were informed that they could not participate or be compensated. Eligible participants took the survey. At the end, these participants were given a code generated on Qualtrics to submit to MTurk for $\$ 3$ of compensation. On average, the survey took participants 14.3 minutes $($ Median $=12.4$ minutes, $\mathrm{SD}=8.5$ minutes).

The study was approved by our university's Institutional Review Board.

\subsection{Survey Design}

In the survey, participants first answered demographic questions on age, gender, computer proficiency, race, education, and military experience. Next, each viewed their randomly assigned video and was subsequently shown the following text:

Now imagine that you are working for a law enforcement agency as the operator of the presented drone system. Your task is to identify, track, and neutralize the vehicles of human traffickers who could harm civilians if not detained. Please note that failure to identify violent criminals such as human traffickers can put innocent civilians' and law enforcement officers' lives at danger. Please answer the following questions assuming the presented operating conditions.

Participants were then asked to reiterate the scenario in their own words to ensure that they understood their task and role as operator.

Next, we evaluated Mayer et al.'s trusting beliefs in the system's ability, integrity, and benevolence [27]. These items were adapted from $[26,30]$ to refer to our drone system (see Table 3 ).

Subsequently, participants answered questions about situational normality and structural assurance. These institutional trust items were adapted from $[25,29]$ to refer to the government law enforcement agency in our hypothetical scenario.

Participants then reported on their propensity to trust other people using a 12-item scale [12] and risk-taking tendencies in 5 domains (financial, ethical, health/safety, recreational, social) using the 30-item domain-specific risk-taking (DOSPERT) scale [4, 18, 40, 43].

We included two questions in the survey asking for a specific answer (e.g., "Mostly Agree") to identify inattentive participants. We also included two manipulation check items at the end of the survey to validate that the system's performance and process were communicated in the video.

\section{EVALUATION}

\subsection{Sample Demographics}

Of the 200 participants eligible after pre-screening, we removed the data of those who incorrectly answered at least one multiple choice attention check question, entered an ineligible age, or misunderstood the scenario based on their post-video reiteration. For the latter, participants who gave an unrelated response, referred to the "operator" in the third-person (i.e., suggesting that they were not imagining being the operator) or mentioned something not expressed in the video (e.g., "the military," "drug sales," "child sex traffickers") were removed from the data. Lastly, to ensure the video was fresh in participants' minds, we removed data of those who waited greater than 10 minutes after their video ended to advance to the next part of the survey. Ultimately, 163 were retained for analysis and balanced among video groups (see Table 1).

The sample consisted of 89 (54.6\%) male and 74 (45.4\%) female participants with ages ranging from 20 to 64 (Mean $=35.3$, SD $=10.0$ ). When asked about computer proficiency, 58 (35.6\%) participants reported being "Competent," 82 (50.3\%) "Proficient," and 23 (14.1\%) "Expert." There were 123 white/Caucasian (75.5\%), 18 African American (11.0\%), 6 Hispanic (3.7\%), 11 Asian (6.7\%), 2 


\begin{tabular}{cl}
\hline Trusting Belief & Items \\
\hline \multirow{3}{*}{ Ability } & - The drone system would be competent and effective at assisting in tracking enemy targets. \\
& - The drone system would perform its role of neutralizing enemy targets very well. \\
& - Overall, the drone system would be a capable and proficient means for stopping the targets. \\
& - In general, the drone system would be very knowledgeable about stopping criminals. \\
\hline & - The drone system would be truthful in its communication with me. \\
- I would characterize the drone system as honest. & - The drone system would keep its commitments. \\
Integrity & - The drone system would be sincere and genuine. \\
& - The drone system would perform as expected. \\
& - I believe that the drone system would operate in my best interest. \\
& - If I required help, the drone system would do its best to help me. \\
- The drone system would be concerned about my well-being, not just its own. & - The drone system would be concerned about the well-being of officers on the ground. \\
Benevolence & - The drone system would be concerned about the well-being of civilians.
\end{tabular}

Table 3: The three trusting beliefs and their subsisting items, scored on a 7-point Likert scale from $1=$ "Strongly Disagree" to 7 = "Strongly Agree." These items were adapted from [26, 30].

Native American and 3 other participants. Furthermore, $82.2 \%$ of participants reported having some post-secondary education at a college or university and 7 (4.3\%) reported having served in the military.

Testing for demographic differences between video groups, we found no significant differences in terms of gender $\left(\chi^{2}(3)=2.19\right.$, $p=.53)$. Moreover, Fisher's Exact Test revealed neither significant differences in terms of race $(p=.51)$ nor military service $(p=.32)$. Kruskal-Wallis tests demonstrated that groups were not significantly different in terms of age $(H(3)=1.73, p=.63)$, education level $(H(3)=0.16, p=.98)$, or computer proficiency $(H(3)=2.60, p$ $=.46$ ). Based on these results, we concluded that the four groups recruited were similar in terms of their demographics.

\subsection{Validation of Information Types Communicated in the Videos}

First, to verify whether performance and process information were communicated in the narration, we included two manipulation check statements at the end of the survey:

1. I was made aware of the drone system's performance (i.e., how effective the system is about accomplishing its goal).

2. I was made aware of the drone system's process (i.e., how the system works to accomplish its goal).

Participants rated these two items on a 7-point Likert scale from "Strongly Disagree" to "Strongly Agree." We use Mann-Whitney U-tests to compare between participants who received or did not receive a given type of information. We also report the effect size of U-tests using $r=Z / \sqrt{N}$ metric [11].

Participants who received performance information in their video (i.e., Performance and Perf-Proc groups) rated their awareness of the drone system's performance higher than other participants (i.e., Control and Process groups), though this difference was only marginally significant $(U=2841.00 ; p=.10 ; r=-.13)$. It may be that because the performance information mentioned potential system errors, these participants actually felt somewhat unaware of the system's performance.
Participants who received process information in their video (i.e., Process and Perf-Proc groups) rated their awareness of the system's process significantly higher than other participants (i.e., Control and Performance groups) $(U=2667.50 ; p=.02 ; r=-.18)$.

\subsection{Building Multiple Linear Regression Models for Perceived Ability, Integrity, and Benevolence}

To answer our research questions, we constructed multiple linear regression models that predict participants' initial trustworthiness perceptions in the system's ability, integrity, and benevolence from a set of input predictors.

Before building models, we verified the reliability of our independent and dependent scales using Cronbach's $\alpha$. The 12-item propensity to trust scale had excellent reliability $(\alpha=.95)$. Each of the DOSPERT 6-item risk domain scales had at least acceptable reliability (ethical $\alpha=.80$; financial $\alpha=.81$; health/safety $\alpha=.71$; recreational $\alpha=.81$; social $\alpha=.74$ ). For institutional trust, each 3 -item situational normality sub-scale had good reliability (ability $\alpha=.88$; integrity $\alpha=.94$; benevolence $\alpha=.87$ ), and the 4 -item structural assurance scale had excellent reliability $(\alpha=.95)$. The three trusting belief scales had good reliability (4-item, ability $\alpha=.81$; 5-item, integrity $\alpha=.88 ; 5$-item, benevolence $\alpha=.85$ ). Overall, both our independent and dependent variables demonstrated acceptable reliability [14].

Next, we considered predicting each perceived trustworthiness characteristic with a different set of independent variables. For each model, we examined the Bayesian Information Criterion (BIC), a goodness-of-fit metric which also takes into account the model's complexity. A lower relative value suggests better fit for a given model.

We first included only the experimental manipulations as predictors, using performance and process variables to indicate whether each information type was presented in a participant's video. For example, the regression coefficient for the performance variable compares trustworthiness ratings given by participants for whom 


\begin{tabular}{|c|c|c|c|c|c|c|c|c|c|}
\hline \multirow[b]{2}{*}{ Predictors } & \multicolumn{3}{|c|}{ Ability } & \multicolumn{3}{|c|}{ Integrity } & \multicolumn{3}{|c|}{ Benevolence } \\
\hline & $\beta$ & $S E$ & $p$ & $\beta$ & $S E$ & $p$ & $\beta$ & $S E$ & $p$ \\
\hline \multicolumn{10}{|l|}{ Individual Differences } \\
\hline Trusting propensity & -0.16 & 0.08 & 0.04 & -0.02 & 0.12 & 0.90 & -0.15 & 0.13 & 0.27 \\
\hline \multicolumn{10}{|l|}{ Risk-taking: } \\
\hline Ethical & -0.02 & 0.07 & 0.73 & 0.09 & 0.10 & 0.41 & 0.18 & 0.12 & 0.14 \\
\hline Financial & 0.13 & 0.05 & 0.02 & 0.23 & 0.08 & $<0.01$ & 0.32 & 0.09 & $<0.01$ \\
\hline Health/safety & -0.12 & 0.07 & 0.09 & 0.00 & 0.10 & 0.99 & -0.08 & 0.12 & 0.50 \\
\hline Recreational & -0.01 & 0.06 & 0.83 & -0.13 & 0.09 & 0.12 & -0.04 & 0.10 & 0.71 \\
\hline Social & 0.05 & 0.06 & 0.35 & -0.09 & 0.08 & 0.30 & -0.15 & 0.10 & 0.12 \\
\hline \multicolumn{10}{|l|}{ System Information } \\
\hline Perf & 0.34 & 0.17 & 0.04 & 0.29 & 0.24 & 0.23 & 0.35 & 0.28 & 0.22 \\
\hline Proc & 0.37 & 0.17 & 0.03 & 0.55 & 0.25 & 0.03 & 0.57 & 0.29 & 0.05 \\
\hline Perf x Proc & -0.36 & 0.23 & 0.13 & -0.57 & 0.34 & 0.10 & -0.53 & 0.40 & 0.18 \\
\hline \multicolumn{10}{|l|}{ Institutional Trust } \\
\hline Structural Assurance & 0.38 & 0.11 & $<0.01$ & 0.45 & 0.16 & $<0.01$ & 0.42 & 0.19 & 0.03 \\
\hline \multicolumn{10}{|l|}{ Situational Normality: } \\
\hline Ability & 0.12 & 0.12 & 0.32 & 0.20 & 0.18 & 0.26 & 0.20 & 0.21 & 0.33 \\
\hline Integrity & 0.13 & 0.11 & 0.23 & -0.21 & 0.16 & 0.19 & -0.25 & 0.18 & 0.18 \\
\hline Benevolence & -0.09 & 0.12 & 0.42 & 0.13 & 0.17 & 0.44 & 0.19 & 0.20 & 0.36 \\
\hline Constant & 2.65 & 0.45 & $<0.01$ & 1.81 & 0.65 & $<0.01$ & 1.29 & 0.76 & 0.09 \\
\hline Adjusted $R^{2}$ & \multicolumn{3}{|c|}{0.4888} & \multicolumn{3}{|c|}{0.3254} & \multicolumn{3}{|c|}{0.2623} \\
\hline F-value & \multicolumn{3}{|c|}{$F(13,149)=12.92(p<.001)$} & \multicolumn{3}{|c|}{$F(13,149)=7.01(p<.001)$} & \multicolumn{3}{|c|}{$F(13,149)=5.43(p<.001)$} \\
\hline
\end{tabular}

Table 4: Results of the three separate multiple linear regressions, each predicting a trusting belief (ability, integrity, or benevolence) based on the various predictors. $p$-values which are significant at the 0.05 level are shown in bold.

performance information was present (i.e., Performance and PerfProc groups) to those for whom it was absent (i.e., Control and Process groups). The BIC value was 487.79 for the ability regression, 567.81 for integrity, and 601.51 for benevolence. Next, we added our other independent variables to each model to observe whether there was improvement in predicting perceived trustworthiness. Specifically, each trusting belief was regressed on dispositional factors of the participant (i.e., trusting propensity, risk-taking domains), situational factors that captured participants' perceptions of the hypothetical law enforcement agency behind the system (i.e., situational normality, structural assurance), and the aforementioned learned factors (i.e., system information provided in the videos). The BIC value was 418.45 for ability, 543.25 for integrity, and 592.10 for benevolence, indicating that the fit of the models improved with the addition of dispositional and situational factors. The results of these final regressions are shown in Table 4.

\subsubsection{Dispositional Trust.}

Regarding RQ1, participants with a greater propensity to trust other people were more likely to rate the system's ability lower $(\beta=-0.16, p<.05)$. This suggests that individuals who are more trusting of other people have less faith in the ability of the drone system. We return to the influence of trusting propensity below when reporting on institutional (i.e., situational) trust.

For RQ2, individuals who reported greater financial risk-taking tendencies were likely to give higher ratings for the system's ability ( $\beta=0.13, p<.05)$, integrity $(\beta=0.23, p<.01)$, and benevolence
( $\beta=0.32, p<.01)$. These findings support those in [20] regarding both the role of individual differences in dispositional trust, as well as the distinction between dispositional trust in humans and in automation.

\subsubsection{Learned Trust.}

Concerning RQ3, performance and process information given to participants appeared to influence ability and integrity beliefs.

Interestingly, despite the mention of potential system errors, participants receiving performance information were likely to rate the system's ability higher than those not receiving performance information $(\beta=0.34, p<.05)$. While this suggests that transparency increases perceptions of trustworthiness, the fact that users knew about errors and regarded the system as having higher ability is a poor sign for trust calibration. System designers should be explicit about their system's weaknesses, not necessarily aiming to increase trust but to promote appropriate calibration. Moreover, while our finding suggests a connection between performance information and increased ability beliefs, it may be that perceptions of system ability as measured in this study do not necessarily lead to increased behavioral trust (i.e., greater reliance on the system). Information about system errors may actually make participants less willing to use the system in certain situations despite higher trusting belief ratings.

Process information appeared to lead to increased perceptions of ability $(\beta=0.37, p<.05)$ and integrity $(\beta=0.55, p<.05)$. Again, this lends to the idea that people appreciate transparency-knowledge 
about the system's underlying technologies seems to have led participants to more positively regard its ability and integrity.

Despite these findings, the interaction between performance and process information did not significantly influence any of the trusting beliefs. This suggests that a larger volume of information does not necessarily contribute positively to trusting beliefs.

\subsubsection{Situational Trust.}

For RQ4, situational normality ratings did not have a significant effect on perceived trustworthiness. However, participants who gave higher structural assurance ratings were likely to give higher ratings for the ability $(\beta=0.38, p<.01)$, integrity $(\beta=0.45, p<.01)$, and benevolence $(\beta=0.42, p<.01)$ of the drone system. This finding demonstrates the important distinction between perceptions of the system itself and of the institution behind the system. While we find that positive beliefs about institutional safeguards contributed to more positive perceptions of the system's trustworthiness characteristics, the contributions from various other factors in our model show that institutional trust is not the full picture of trust in the system.

To explore structural assurance more in depth, we ran a multiple linear regression with structural assurance as the dependent variable and the same set of independent variables (excluding institutional trust items). A significant regression equation was found $(F(9,153)=2.86, p<.01)$ with an Adjusted $R^{2}$ of 0.0936 . The only significant independent factor was propensity to trust, where more trusting participants were likely to give higher structural assurance ratings $(\beta=0.49, p<.01)$. This effect on structural assurance suggests that participants' interpersonal trust indirectly affected trustworthiness perceptions of the drone system through their perception of the law enforcement agency.

\section{DISCUSSION}

Our findings suggest that users' individual differences, information about a system, and institutional perceptions each contribute differently to initial beliefs about a system's ability, integrity, and benevolence. The key findings and limitations of our study are discussed below.

\subsection{Effect of Risk-Taking Tendencies on Ability, Integrity, and Benevolence Beliefs}

We found that individuals who reported being more likely to take financial risks rated the system's ability, integrity, and benevolence more highly. Interestingly, the largest effect (i.e., greatest regression coefficient) was on the benevolence belief, followed by integrity and, subsequently, ability. This finding lends support to our application of Mayer et al.'s trustworthiness characteristics to an automated trustee, in that each was influenced differently by financial risk-taking tendencies. For instance, the fact that benevolence beliefs were the most influenced by dispositional characteristics suggests that users did build some idea of whether the hypothetical drone system cared about them, based partly on their willingness to take risks (see "Benevolence" items in Table 3), even if it may seem inappropriate to think of a computer system as having the quality of benevolence. While this benevolence perception may reflect participants' thoughts about the institution's intentions, further investigating this phenomenon by applying the "Computers are Social Actors" paradigm could elucidate the nature of human perceptions of automated trustees. We encourage future work to further explore this relationship between individual differences and the trustworthiness characteristics to inform more user-centered designs for trust calibration.

\subsection{Effect of System Information on Ability and Integrity Beliefs}

Our regression also revealed that performance or process information in the narration was likely to lead to increased ability beliefs. Moreover, process information was likely to lead to increased integrity beliefs. We note that although our manipulation check revealed that those who received performance information reported being more aware of the system's performance than those who did not receive performance information, the difference was not significant. Nonetheless, the performance and process information (see Table 2) appear to have offered transparency, casting the system as less of a fault-prone black box. Given performance information, the system may have appeared more competent because its shortcomings were directly acknowledged. For process information, the description of the system's underlying technologies (i.e., flight stability algorithms, network connection to the operator) may have contributed to these increased perceptions of trustworthiness.

In a prior study of an autonomous driving system, Koo et al. observed that a "how" explanation (i.e., reflecting the system's process) led to more drivers drifting out of their lane [21]. Their "how" explanation, "The car is braking," may have caused reliance on the system in inappropriate circumstances (i.e., overtrust and misuse [35]) by insufficiently communicating limitations of the system to its user. While our process information also increased participants' perceptions of system ability and integrity, an important question is how these perceptions ultimately impact users' behaviors with the system, though this is outside of our focus on initial trust. We encourage future work to investigate how initial trust influences long-term interactions and trust calibration with safety-critical systems.

In another similar study, drivers who were shown performance information about an autonomous driving system were found to have lower trust levels than those not shown the information, and were more prepared to take over control from the automation [15]. This suggests that the information allowed for trust calibration. Likewise, take-over requests and alerts have been found to lead to safer usage and greater trust [16, 42]. While our information increased users' perceptions of system trustworthiness, a more appropriate goal for system designers and institutions is to give information that improves trust. In some cases, this may mean giving trust-reducing information that allows users to calibrate to a particular system's shortcomings. While our performance information could be seen as trust-reducing, those who received this information rated the system's ability higher than those who did not receive it. This is likely a result of ambiguity regarding the extent to which system errors would interfere with operation of the system in our task context.

While aforementioned prior work manipulates trust during interaction with the system, we observe users' initial impressions. Although this makes it more difficult to compare our results to 
prior efforts, it allows us to isolate the effect of system information on learned trust, as participants were not affected by use-related factors such as interface design and observed system performance. We encourage future work to explore the specific role of trusting beliefs in users' reliance decisions. For example, it may be that beliefs about integrity are less critical to reliance on a system than those about its ability.

The insignificant effect of the interaction between performance and process information suggests that the mention of system errors alongside a description of the system's underlying technologies tempered trustworthiness ratings compared to either information type given on its own. In this way, trust calibration may essentially be a balancing act, as suggested in de Visser et al.'s model involving trust repair acts and trust dampening acts [8]. Safety-critical systems can incorporate communication modules that relay information to users following both system malfunctions and successful operations, in order to mitigate large changes in a user's perceptions of system trustworthiness and ensure that appropriately calibrated trust is maintained.

\subsection{Effect of Institutional Trust on Ability, Integrity, and Benevolence Beliefs}

We found that participants who felt more structurally assured were likely to have higher initial beliefs in the system's ability, integrity, and benevolence. We also found that trusting propensity contributed to greater perceptions of structural assurance. Although this implies that trusting individuals will be more trusting of a safety-critical system, overtrust is not desirable and can lead to use of the system in inappropriate, dangerous circumstances. Institutions that oversee safety-critical operations must carefully create structural assurances such that users are held appropriately accountable for misuse of the system (i.e., to avoid overtrust), but not necessarily for failures caused by the system itself.

\subsection{Limitations}

Though the present study sheds light on factors in initial trust of safety-critical systems, the reported findings should be interpreted with caution due to the following limitations of our study.

First, participants had to imagine being the operator without actually interacting with a system. Because of this, their perception of risk may have been diminished. The study also uses self-reported trust ratings which, although indicative of users' initial perceptions, were not connected to a behavioral measure. Nonetheless, our study does confirm the effect of different factors on the three perceived trustworthiness characteristics, which was the main objective of our work. Further research is needed to confirm our findings and measure the effect of initial trusting beliefs on actual system interaction over time in controlled lab settings, possibly using experimental deception to create greater perceived risk. Future work should also look at the influence of system information on trust formation in systems with different reliability levels, since trust has often been found to vary with respect to system reliability [19], and appropriate calibration of trust is our main goal.

Second, since this was an online survey, we could not guarantee that participants remained attentive during the video. It is possible that some MTurk participants were multitasking and did not pay attention to the narrated information. However, to minimize this possibility, each participant had to wait at least their video duration before proceeding and we removed data of participants whose open-ended responses demonstrated a lack of understanding of the task.

Finally, there are some limitations to our manipulations. Due to differences in narrated content, the videos were not exactly the same length. For one, this caused longer videos to contain more visual content than the shorter videos. To minimize any possible effect of visual content, we carefully chose the shots of the drone control panel and operators to be relatively neutral, especially compared to the safety-critical task described in the narration. Moreover, receiving a greater amount of information could have contributed to participants' differences in perception. However, we tested for correlation between video length and trusting belief ratings and found no significant correlations, suggesting that informational content is what influenced perceived trustworthiness.

We also acknowledge potential unintended effects of message content. In particular, the performance information discussed "occasional errors" and may have been perceived more negatively than the process information. Studying the effects of valence of system information (e.g., positive performance information vs. negative performance information) may give further insights. Likewise, interpretation of the phrase "such failures" in the process information may have been interpreted differently depending on whether or not a participant received performance information. Our manipulation check did suggest that awareness of system's performance and process was increased by the narrated information types. However, we encourage future research to build upon these initial findings to better understand various informational contributors to trustworthiness perceptions.

\section{CONCLUSION}

In this study, we investigated the role of dispositional, learned, and situational factors on initial trustworthiness perceptions. We designed a control video with information about a hypothetical drone system and three videos with either additional performance or process information, or both, to observe how 163 naive participants on Amazon's MTurk formed trusting beliefs based on the video. We found that risk-taking individual differences affected all three beliefs. Moreover, performance and process information were likely to increase beliefs in the system's ability, while process information also lead to increased integrity beliefs. We stress that increased trust is not necessarily desirable-users should instead be able to calibrate their trust in a system to an appropriate level. Lastly, we found that structural assurance was associated with increased ability, integrity, and benevolence perceptions. We believe that insights gained from this study enhance our understanding of user's multidimensional perceptions of the "trustworthiness" of safety-critical systems, which can lead to more favorable trust-related outcomes of human-computer collaborations.

\section{ACKNOWLEDGMENTS}

This material is based upon work supported by the Air Force Office of Scientific Research under award number FA9550-15-1-0490. 


\section{REFERENCES}

[1] Bernard Barber. 1983. The logic and limits of trust. (1983).

[2] Izak Benbasat and Weiquan Wang. 2005. Trust in and adoption of online recommendation agents. Fournal of the association for information systems 6, 3 (2005), 4.

[3] David P Biros, Mark Daly, and Gregg Gunsch. 2004. The influence of task load and automation trust on deception detection. Group Decision and Negotiation 13, 2 (2004), 173-189.

[4] Ann-Renée Blais and Elke U Weber. 2006. A domain-specific risk-taking (DOSPERT) scale for adult populations. (2006).

[5] Ross Buck. 2014. Emotion: A biosocial synthesis. Cambridge University Press.

[6] Michelle S Carlson, Munjal Desai, Jill L Drury, Hyangshim Kwak, and Holly A Yanco. 2014. Identifying factors that influence trust. In 2014 AAAI Spring Symposium Series.

[7] James M Clark and Allan Paivio. 1991. Dual coding theory and education. Educational psychology review 3, 3 (1991), 149-210.

[8] Ewart J de Visser, Richard Pak, and Tyler H Shaw. 2018. From "automation" to "autonomy": The importance of trust repair in human-machine interaction. Ergonomics just-accepted (2018), 1-33.

[9] Morton Deutsch. 1960. Trust, trustworthiness, and the F scale. The fournal of Abnormal and Social Psychology 61, 1 (1960), 138.

[10] Stephen R Dixon, Christopher D Wickens, and Dervon Chang. 2004. Unmanned aerial vehicle flight control: False alarms versus misses. In Proceedings of the Human Factors and Ergonomics Society Annual Meeting, Vol. 48. SAGE Publications Sage CA: Los Angeles, CA, 152-156.

[11] Andy Field. 2013. Discovering statistics using IBM SPSS statistics. Sage.

[12] M Lance Frazier, Paul D Johnson, and Stav Fainshmidt. 2013. Development and validation of a propensity to trust scale. Fournal of Trust Research 3, 2 (2013), 76-97.

[13] Amos Freedy, Ewart de Visser, Gershon Weltman, and Nicole Coeyman. 2007. Measurement of trust in human-robot collaboration. In Collaborative Technologies and Systems, 2007. CTS 2007. International Symposium on. IEEE, 106-114.

[14] Joseph A Gliem and Rosemary R Gliem. 2003. Calculating, interpreting, and reporting Cronbach's alpha reliability coefficient for Likert-type scales. Mid west Research-to-Practice Conference in Adult, Continuing, and Community Education.

[15] Tove Helldin, Göran Falkman, Maria Riveiro, and Staffan Davidsson. 2013. Presenting system uncertainty in automotive UIs for supporting trust calibration in autonomous driving. In Proceedings of the 5th International Conference on Automotive User Interfaces and Interactive Vehicular Applications. ACM, 210-217.

[16] Sebastian Hergeth, Lutz Lorenz, Josef F Krems, and Lars Toenert. 2015. Effects of take-over requests and cultural background on automation trust in highly automated driving. In 8th International Driving Symposium on Human Factors in Driver Assessment, Training, and Vehicle Design.

[17] Carol Herron, Holly York, Cathleen Corrie, and Steven P Cole. 2006. A comparison study of the effects of a story-based video instructional package versus a textbased instructional package in the intermediate-level foreign language classroom. Calico fournal (2006), 281-307.

[18] Scott Highhouse, Christopher D Nye, Don C Zhang, and Thaddeus B Rada. 2017 Structure of the Dospert: Is There Evidence for a General Risk Factor? Journal of Behavioral Decision Making 30, 2 (2017), 400-406.

[19] Kevin Anthony Hoff and Masooda Bashir. 2015. Trust in automation: Integrating empirical evidence on factors that influence trust. Human Factors 57, 3 (2015), 407-434.

[20] Hsiao-Ying Huang and Masooda Bashir. 2017. Personal Influences on Dynamic Trust Formation in Human-Agent Interaction. In Proceedings of the 5th International Conference on Human Agent Interaction. ACM, 233-243.

[21] Jeamin Koo, Jungsuk Kwac, Wendy Ju, Martin Steinert, Larry Leifer, and Clifford Nass. 2015. Why did my car just do that? Explaining semi-autonomous driving actions to improve driver understanding, trust, and performance. International fournal on Interactive Design and Manufacturing (IfIDeM) 9, 4 (2015), 269-275.

[22] Marios Koufaris and William Hampton-Sosa. 2004. The development of initial trust in an online company by new customers. Information \& management 41,3 (2004), 377-397.

[23] John Lee and Neville Moray. 1992. Trust, control strategies and allocation of function in human-machine systems. Ergonomics 35, 10 (1992), 1243-1270.

[24] John D Lee and Katrina A See. 2004. Trust in automation: Designing for appropriate reliance. Human factors 46, 1 (2004), 50-80.

[25] Xin Li, Traci J Hess, and Joseph S Valacich. 2008. Why do we trust new technology? A study of initial trust formation with organizational information systems. The fournal of Strategic Information Systems 17, 1 (2008), 39-71.

[26] Roger C Mayer and James H Davis. 1999. The effect of the performance appraisal system on trust for management: A field quasi-experiment. fournal of applied psychology 84, 1 (1999), 123.

[27] Roger C Mayer, James H Davis, and F David Schoorman. 1995. An integrative model of organizational trust. Academy of management review 20, 3 (1995), 709-734.

[28] Richard E Mayer and Valerie K Sims. 1994. For whom is a picture worth a thousand words? Extensions of a dual-coding theory of multimedia learning. fournal of educational psychology 86, 3 (1994), 389.

[29] D Harrison McKnight, Vivek Choudhury, and Charles Kacmar. 2002. Developing and validating trust measures for e-commerce: An integrative typology. Information systems research 13, 3 (2002), 334-359.

[30] D Harrison McKnight, Vivek Choudhury, and Charles Kacmar. 2002. The impact of initial consumer trust on intentions to transact with a web site: a trust building model. The fournal of Strategic Information Systems 11, 3 (2002), 297-323.

[31] D Harrison McKnight, Larry L Cummings, and Norman L Chervany. 1998. Initial trust formation in new organizational relationships. Academy of Management review 23, 3 (1998), 473-490.

[32] Bonnie M Muir. 1987. Trust between humans and machines, and the design of decision aids. International fournal of Man-Machine Studies 27, 5-6 (1987), 527-539.

[33] Clifford Nass and Youngme Moon. 2000. Machines and mindlessness: Social responses to computers. Journal of social issues 56, 1 (2000), 81-103.

[34] Clifford Nass, Jonathan Steuer, and Ellen R Tauber. 1994. Computers are social actors. In Proceedings of the SIGCHI conference on Human factors in computing systems. ACM, 72-78.

[35] Raja Parasuraman and Victor Riley. 1997. Humans and automation: Use, misuse, disuse, abuse. Human factors 39, 2 (1997), 230-253.

[36] Darcy Podszebka, Candee Conklin, Mary Apple, and Amy Windus. 1998. Comparison of Video and Text Narrative Presentations on Comprehension and Vocabulary Acquisition. (1998).

[37] Byron Reeves and Clifford Nass. 1996. How people treat computers, television, and new media like real people and places. CSLI Publications and Cambridge university press.

[38] Julian B Rotter. 1967. A new scale for the measurement of interpersonal trust. Journal of personality 35, 4 (1967), 651-665.

[39] Julian B Rotter. 1980. Interpersonal trust, trustworthiness, and gullibility. American psychologist 35, 1 (1980), 1.

[40] Sim B Sitkin and Amy L Pablo. 1992. Reconceptualizing the determinants of risk behavior. Academy of management review 17, 1 (1992), 9-38.

[41] Nadaleen Tempelman-Kluit. 2006. Multimedia learning theories and online instruction. College \& Research Libraries 67, 4 (2006), 364-369.

[42] Remo van der Heiden, Shamsi T Iqbal, and Christian P Janssen. 2017. Priming Drivers before Handover in Semi-Autonomous Cars. In Proceedings of the 2017 CHI Conference on Human Factors in Computing Systems. ACM, 392-404.

[43] Elke U Weber, Ann-Renee Blais, and Nancy E Betz. 2002. A domain-specific risk-attitude scale: Measuring risk perceptions and risk behaviors. Fournal of behavioral decision making 15, 4 (2002), 263-290. 